\title{
A pharmacokinetic binding model for bevacizumab and VEGF 165 in colorectal cancer patients
}

\author{
Eirini Panoilia $\cdot$ Emilie Schindler $\cdot$ Epaminontas Samantas $\cdot$ \\ Gerasimos Aravantinos · Haralabos P. Kalofonos · Christos Christodoulou • \\ George P. Patrinos $\cdot$ Lena E. Friberg $\cdot$ Gregory Sivolapenko
}

Received: 25 November 2014 / Accepted: 10 February 2015 / Published online: 17 February 2015

(C) The Author(s) 2015. This article is published with open access at Springerlink.com

\begin{abstract}
Purpose To characterize the population pharmacokinetics of bevacizumab, its binding properties to $\mathrm{VEGF}_{165}$ and the effect of demographic data and VEGF-A polymorphisms on the interplay between bevacizumab serum pharmacokinetics and $\mathrm{VEGF}_{165}$ serum concentrations in patients with colorectal cancer stage IV.

Methods Bevacizumab and $\mathrm{VEGF}_{165}$ data were collected from 19 adult patients with metastatic colorectal cancer enrolled in an observational clinical study. Bevacizumab was administered with one of the following combinations:
\end{abstract}

Electronic supplementary material The online version of this article (doi:10.1007/s00280-015-2701-3) contains supplementary material, which is available to authorized users.

E. Panoilia $(\varangle) \cdot$ G. P. Patrinos $\cdot$ G. Sivolapenko

Department of Pharmacy, University of Patras, Rio-Patras,

Greece

e-mail: eipanoilia@upatras.gr

E. Schindler · L. E. Friberg

Department of Pharmaceutical Biosciences, Uppsala University,

Uppsala, Sweden

\section{E. Samantas}

3rd Department of Medical Oncology, "Agii Anargiri” Cancer

Hospital, Kalyftaki-Nea Kifissia, Greece

\section{G. Aravantinos}

2nd Department of Medical Oncology, "Agii Anargiri” Cancer

Hospital, Kalyftaki-Nea Kifissia, Greece

\section{H. P. Kalofonos}

Division of Medical Oncology, University Hospital of Patras,

Rio-Patras, Greece

C. Christodoulou

2nd Department of Medical Oncology, "Metropolitan" Hospital,

Athens, Greece
5-FU/Leucovorin/Irinotecan, 5-FU/Leucovorin/Oxaliplatin, Capecitabine/Irinotecan at doses ranging from 5 to $10 \mathrm{mg} / \mathrm{kg}$ every 2 or 3 weeks. Data analysis was performed using nonlinear mixed-effects modeling implemented in NONMEM 7.3.

Results A target-mediated drug disposition model adequately described bevacizumab concentration changes over time and its binding characteristics to $\mathrm{VEGF}_{165}$. The estimated clearance of bevacizumab was $0.18 \mathrm{~L} /$ day, the free VEGF $_{165}$ levels at baseline were $212 \mathrm{ng} / \mathrm{L}$, and the elimination rate constant of free $\mathrm{VEGF}_{165}$ was $0.401 \mathrm{day}^{-1}$. Body weight was allometrically included in all PK parameters.

Conclusion The final model adequately described the preand post-dose concentrations of total bevacizumab and free VEGF $_{165}$ in patients with colorectal cancer. Model parameters were consistent with those previously reported for patients with solid tumors. Correlations between the binding affinity of bevacizumab and the VEGF-2578C/A and VEGF-634G/C polymorphisms were noticed.

Keywords Bevacizumab - VEGF - Population modeling · VEGF polymorphisms $\cdot$ Colorectal cancer

\section{Introduction}

Bevacizumab, the active substance of Avastin, is a recombinant humanized IgG1 monoclonal antibody with multiple cancer indications. It has been approved in combination with chemotherapy for the treatment of metastatic colorectal cancer (CRC), metastatic breast cancer, unresectable advanced, metastatic or recurrent non-small cell lung cancer, advanced or metastatic renal cell cancer, advanced epithelial cancer of the ovary, the fallopian tube or the peritoneum and as a single agent for advanced glioblastoma [1-3]. 
Bevacizumab targets circulating vascular endothelial growth factor (VEGF or else VEGF-A), a vasculogenesis and angiogenesis regulator that is overexpressed in most human tumors. By blocking VEGF-A binding to its receptors (VEGFR-1 and VEGFR-2) on the surface of endothelial cells, bevacizumab inhibits tumor angiogenesis, growth and metastases [1, 3-6].

There are five major isoforms of the human VEGF-A glycoprotein $\left(\mathrm{VEGF}_{121}, \mathrm{VEGF}_{145}, \mathrm{VEGF}_{165}, \mathrm{VEGF}_{189}\right.$, $\mathrm{VEGF}_{206}$ ) produced by the alternative splicing of the VEGF-A gene. All isoforms differ in the amino acid length and the binding ability to heparin and heparin sulfate proteoglycans found on the cell surface or in the extracellular matrix. $\mathrm{VEGF}_{145}, \mathrm{VEGF}_{189}$ and $\mathrm{VEGF}_{206}$ are tightly bound to the cell surface and the extracellular matrix, $\mathrm{VEGF}_{121}$ is freely diffusible, and $\mathrm{VEGF}_{165}$ is moderately diffusible. VEGF $_{165}$ is the most abundant and potent stimulator of angiogenesis compared to the other isoforms. It is overexpressed in several cancer types and is related to the ability of a tumor to grow, invade and spread [7, 8].

Several clinical trials in CRC patients indicate an inverse relationship between the concentration of VEGFA in serum or tumor tissue and the clinical outcome [911]. Furthermore, certain VEGF-A polymorphisms have been associated with altered VEGF-A production or promoter activity, causing variability in response to treatment [12-14]. The VEGF-2578CC, VEGF-1154GG and VEGF634CC genotypes have been correlated with higher VEGFA production than other genotypes for VEGF-2578C/A, VEGF-1154G/A and VEGF-634G/C polymorphisms [13, 15]. Thus, free serum VEGF-A levels could serve as a useful biomarker for reflecting the anti-angiogenic activity of bevacizumab and for indicating when dose adjustments are needed to achieve sufficient VEGF-A blockade.

Monoclonal antibodies exhibit more complex concentration-time [pharmacokinetic (PK)] and effect-concentration [pharmacodynamic (PD)] characteristics than small molecules. The main elimination pathway is proteolytic catabolism throughout the body (linear, nonspecific clearance) and not hepatic metabolism or renal excretion. However, the disposition of monoclonal antibodies is often influenced by their high affinity binding to their molecular targets and subsequent degradation of the monoclonal antibody-target complexes via fluid phase or receptormediated endocytosis. This phenomenon is known as target-mediated drug disposition (TMDD), and it usually leads to nonlinear, saturable distribution and elimination $[16,17]$. In recent years, population PKPD modeling has proven to be a useful tool to elucidate the dose-response relationships, to explain inter-individual variability in the observed drug exposure or response and to guide dose selection to achieve the optimal benefit-risk ratio for a given anticancer treatment [18-20].
TMDD modeling has been generally used to describe the dynamics of the drug-target interaction, as it can provide valuable information on the mechanism of the underlying PKPD relationship [17, 21, 22]. The full TMDD model is a complex system of differential equations describing the concentrations of the free drug, the free target and the drug-target complex. Model parameters may not be identifiable when limited experimental data on target, total and free drug concentrations are available. In that case, simplifications of the full TMDD model with fewer parameters can be applied such as the quasi-equilibrium, quasi-steady-state (QSS) and Michaelis-Menten approximation [23, 24].

The full TMDD model is based on the following assumptions: (1) the drug-target binding is a simple, one-to-one binding process, (2) the drug can only bind to its specific molecular target, (3) the drug-target binding takes place in the central and not in the peripheral or depot compartments, (4) only the free drug can distribute into the peripheral compartment, (5) the drug-target complex is totally eliminated, and (6) the free target production and degradation rates are constant and independent of the drug or target concentrations. The main assumption of the QSS approximation, which differentiates it from the full TMDD model or other TMDD approximations, is that the change in the drug-target complex concentration is negligible on the time scale of the other system processes. The free drug, the free target and the drug-target complex are assumed to be in a QSS. The QSS approximation is usually preferred when only total drug and target concentrations are available and is applied for drugs with fast binding, dissociation and internalization rates [22-24].

The aim of the present study was to describe the PK of bevacizumab, its binding properties to $\mathrm{VEGF}_{165}$ and the effect of individual patient characteristics on the relationship between bevacizumab and $\mathrm{VEGF}_{165}$ in patients with stage IV CRC using a population modeling approach. To the best of our knowledge, a limited number of population PK studies for bevacizumab have been conducted so far $[25,26]$. Moreover, little quantitative pharmacology research has been performed to establish the impact of VEGF-A polymorphisms and other relevant covariates on treatment response [27-29]. Therefore, it is anticipated that the development of a binding model for bevacizumab in patients with metastatic CRC would yield a better mechanistic understanding of the biological system, as it could characterize the in vivo interaction of the drug with its soluble target, $\mathrm{VEGF}_{165}$. It would further elucidate the PK characteristics of the drug, its effect on the free target time course and provide more information on target affinity and the influence of covariates (e.g., genetics). 


\section{Materials and methods}

Patients and study design

Nineteen subjects were enrolled in this observational (non interventional) study. Eligible patients had CRC stage IV documented by histology (biopsy through colonoscopy or surgery), CT scans (thoracic, abdominal and brain scans), bone scintiscans and the measurement of cancer biomarkers (CEA and CA 19.9); Eastern Cooperative Oncology Group (ECOG) performance status $\leq 2$; no chemotherapy or radiotherapy within 3 months of first study treatment; were at least 18 years of age. Prior to enrollment, all patients had undergone a complete history and physical examination. Standard hematologic and biochemical laboratory tests had also been conducted to assess an adequate bone marrow, liver and renal function as defined by: white blood cells count $(\mathrm{WBC}) \geq 2500 / \mu \mathrm{L}$, absolute neutrophil count $($ ANC $) \geq 1500 / \mu \mathrm{L}$, platelets $\geq 75,000 / \mu \mathrm{L}$, serum creatinine $\leq 1.5 \times$ upper limit of normal (ULN), urine protein $\leq 2 \mathrm{~g} / 24 \mathrm{~h}$, total serum bilirubin $\leq 3 \mathrm{mg} / \mathrm{dL}$, AST (SGOT)/ALT (SGPT) $\leq 2 \times$ ULN. The research was conducted in accordance with the Declaration of Helsinki, and all the appropriate approvals were obtained by the relevant Ethics Committees. Signed informed consent was provided by all participants before the initiation of the study.

The current study was carried out in three Greek oncology clinics, at the Department of Medical Oncology of Agii Anargiri Cancer Hospital, at the Department of Medical Oncology of Metropolitan Hospital in Athens and at the Division of Oncology of Patras University Hospital in Rio.

Bevacizumab (Avastin ${ }^{\circledR}$, Roche Registration Ltd.) was administered as an intravenous infusion at a dose of $5 \mathrm{mg} /$ $\mathrm{kg}$, in combination with 5-Fluorouracil/Leucovorin/Irinotecan (BEV-FOLFIRI treatment) or 5-Fluorouracil/Leucovorin/Oxaliplatin (BEV-FOLFOX treatment), in 2-week cycles or at a dose of $7.5 \mathrm{mg} / \mathrm{kg}$ together with Capecitabine/Irinotecan (BEV-CAPIRI treatment) in 3-week cycles. One patient received the BEV-FOLFIRI treatment at a dose of $10 \mathrm{mg} / \mathrm{kg}$ in 2-week cycles. The duration of first infusion was $90 \mathrm{~min}$. If no infusion-related symptoms were observed, subsequent infusions were given over 60 or $30 \mathrm{~min}$

Subjects on BEV-FOLFIRI or BEV-FOLFOX treatment received initially six cycles of bevacizumab and those who responded to treatment, as defined by Response Evaluation Criteria in Solid Tumors [30], continued with the same treatment for other six cycles. The responders, after the completion of 12 cycles in total, continued with bevacizumab monotherapy till disease progression. In that case, they were administered bevacizumab at a dose of $5 \mathrm{mg} / \mathrm{kg}$ every 2 weeks.
Subjects on BEV-CAPIRI treatment received initially three or four cycles of bevacizumab (the number of cycles depends on the lines of treatment the patients had undergone before the initiation of the current treatment). After the completion of eight or nine cycles, the responders continued either with the same treatment or bevacizumab monotherapy upon development of disease progression at the recommended dose $(7.5 \mathrm{mg} / \mathrm{kg}$ every 3 weeks).

Pre- and post-dose (after the end of infusion) concentrations of total bevacizumab (free and bound to one molecule of $\mathrm{VEGF}_{165}$ ) and free $\mathrm{VEGF}_{165}$ (unbound to bevacizumab) were measured in serum during several cycles of treatment. Two blood samples (one pre-dose and one post-dose) were drawn on day 1 of the following cycles: (1) 3, 6, 8, 12, 18 and 24 (patients on BEV-FOLFIRI or BEV-FOLFOX treatment), (2) 2, 4, 5, 8 and 11 (patients on BEV-CAPIRI treatment). One pre-dose blood sample was also collected on day 1 of cycle 1 , intended only for free $\mathrm{VEGF}_{165}$ analysis. The sample collection schedule for total bevacizumab and free VEGF $_{165}$ measurements is shown in Supplemental Fig. 1. The genotypes for VEGF-2578C/A, VEGF1154G/A and VEGF-634G/C single-nucleotide polymorphisms (SNPs) were also determined in blood.

\section{Measurement of total bevacizumab in serum}

Blood samples were collected in serum separator tubes and were allowed to clot for $30 \mathrm{~min}$. After centrifugation at $1000 \times g$ for $20 \mathrm{~min}$, the serum was removed and stored in aliquots at $\leq-20^{\circ} \mathrm{C}$ until analysis.

The concentration of total (free and bound to one molecule of $\mathrm{VEGF}_{165}$ ) bevacizumab in serum was measured using a previously published enzyme-linked immunosorbent assay (ELISA), where the detection limit was $0.033 \mathrm{mg} / \mathrm{L}$ and the range of linearity was between 5 and $75 \mathrm{mg} / \mathrm{L}$ with precision $5.6 \%$ [expressed as coefficient of variation $(\mathrm{CV})$ percentage]. Standards of $0.24,0.47,0.94$, $1.88,3.75,7.5,15$ and $30 \mathrm{mg} / \mathrm{L}$ were used to generate the standard curve, which are well above the detection limit of the assay and within the range of linearity [31].

Microtiter Nunc Maxisorp 96-well plates were coated with recombinant human $\mathrm{VEGF}_{165}$ (R\&D Systems ${ }^{\circledR}$ Europe) at a concentration of $0.15 \mathrm{mg} / \mathrm{L}$ in carbonate-bicarbonate buffer (1 M, pH 9.6) overnight at $4{ }^{\circ} \mathrm{C}(100 \mu \mathrm{L} /$ well $)$. After washing four times with phosphate-buffered saline (PBS) containing $0.05 \%$ Tween 20 , the wells were blocked with PBS containing $1 \%$ BSA (200 $\mu \mathrm{L} /$ well) and were incubated for $2 \mathrm{~h}$ at room temperature. Afterward, the plates were washed and $100 \mu \mathrm{L}$ of 1:100 diluted standards and samples in $1 \%$ PBS-BSA was added and were incubated for $1 \mathrm{~h}$ at $37{ }^{\circ} \mathrm{C}$ in an incubator shaker. Then, the plates were washed again, and $100 \mu \mathrm{L}$ of peroxidase-conjugated goat antihuman IgG specific for Fc fragment (AbD Serotec ${ }^{\circledR}$, A 
Bio-Rad Company) diluted in $1 \%$ PBS-BSA was added to each well. After 1-h incubation at room temperature followed by washing, $100 \mu \mathrm{L}$ OPD (Sigma-Aldrich) was added and the reaction was allowed to develop at room temperature in the dark. The color reaction was stopped with the addition of sulfuric acid $(2 \mathrm{M}, 50 \mu \mathrm{L} /$ well).

The optical density was measured at $450 \mathrm{~nm}$ with a correction at $650 \mathrm{~nm}$ using an ELISA plate reader (ThermoMax, Molecular Devices). Duplicate readings for 1:100 diluted standards and samples were performed.

The best fit line of the standard curve was determined by regression analysis using OriginPro 8.0 software (Origin$\mathrm{Lab}^{\circledR}$ Corporation). The concentrations read from the standard curve were multiplied by the dilution factor.

Measurement of free $\mathrm{VEGF}_{165}$ in serum

Blood samples were collected in serum separator tubes and were allowed to clot for $30 \mathrm{~min}$. After centrifugation at $1000 \times g$ for $20 \mathrm{~min}$, the serum was removed and stored in aliquots at $\leq-20^{\circ} \mathrm{C}$ until analysis.

The concentration of free $\mathrm{VEGF}_{165}$ (unbound to bevacizumab) in serum was measured by a commercially available ELISA kit for VEGF $_{165}$ (Quantikine ${ }^{\circledR}$ human VEGF, R\&D Systems ${ }^{\circledR}$ Europe). The detection limit of the assay was $9 \mathrm{ng} / \mathrm{L}$, and the precision was $6.7 \%$ (CV \%) [32]. According to the manufacturer, this ELISA assay has not been tested yet for interference with the detection of free or total (free and bound to bevacizumab) $\mathrm{VEGF}_{165}$ in the presence of bevacizumab. To confirm the hypothesis that it can only discriminate and quantitate free $\mathrm{VEGF}_{165}$, we measured $\mathrm{VEGF}_{165}$ concentrations in samples after the addition of increasing concentrations of bevacizumab. $\mathrm{VEGF}_{165}$ standards (1000 and $250 \mathrm{ng} / \mathrm{L}$, respectively) were mixed with increasing $\mathrm{VEGF}_{165}$-to-bevacizumab molar ratios of 1:0, 1:0.1, 1:1 and 1:1000.

The assay procedure is briefly described below. Plates pre-coated with a mouse anti-VEGF antibody were used to capture $\mathrm{VEGF}_{165}$ in standards or samples. Any unbound proteins were washed off and a peroxidase-conjugated polyclonal antibody specific for $\mathrm{VEGF}_{165}$ was added. Then, the plates were washed again and tetramethylbenzidine substrate solution was added. A blue color was developed in proportion to the amount of $\mathrm{VEGF}_{165}$ present in the ELISA samples. Color development was stopped with the addition of sulfuric acid.

The optical density was measured at $450 \mathrm{~nm}$ with a correction at $550 \mathrm{~nm}$ using an ELISA plate reader (ELx800 ${ }^{\mathrm{TM}}$, BioTek Instruments). All standards' and samples' readings were performed in duplicate.

A standard curve was generated with $\mathrm{VEGF}_{165}$ concentrations ranging from 31.2 to $2000 \mathrm{ng} / \mathrm{L}$. The best fit line was determined by regression analysis using OriginPro 8.0 software (OriginLab ${ }^{\circledR}$ Corporation).
VEGF genotyping

Genomic DNA was isolated from blood $(3 \mathrm{~mL})$ using the Gentra Puregene Blood kit (QIAGEN). DNA concentrations were determined by measuring the optical density at $260 \mathrm{~nm}$ with a UV-Vis spectrophotometer (NanoDrop 2000, Thermo Fisher Scientific). DNA purity, which is indicated by the ratio of optical density at 260 and $280 \mathrm{~nm}$, was 1.7-1.9.

VEGF polymorphisms (-2578C/A (rs699947), $-1154 \mathrm{G} / \mathrm{A}$ (rs1570360) and -634G/C (rs2010963) on VEGF genomic DNA) were analyzed by polymerase chain reaction (PCR) using the KAPA2G Fast HotStart ReadyMix kit (Kapa Biosystems, fidelity of the DNA polymerase: 1 error per $1.7 \times$ $10^{5}$ nucleotides incorporated). The primers VEGF-1154 For 5'-TTCAGGCTGTGAACCTTGG-3', VEGF-1154 Rev 5'-G GGCGGTGTCTGTCTGTC-3', VEGF-634 For 5'-TTCAGG CTGTGAACCTTGG-3', VEGF-634 Rev 5'-GGGCGGTGTC TGTCTGTC-3', VEGF-2578 For 5'-AGCAACATGTGCTGA GGATG-3' ${ }^{\prime}$, VEGF-2578 Rev 5'-CCCTTTTCCTCCAACTC TCC-3' were used to amplify fragments of the VEGF gene.

PCR was done with 40 cycles at $95{ }^{\circ} \mathrm{C}$ for $2 \mathrm{~min}$, at $60-65{ }^{\circ} \mathrm{C}$ (VEGF-1154G/A and VEGF-634G/C) or $63{ }^{\circ} \mathrm{C}$ (VEGF-2578C/A) for $15 \mathrm{~s}$ and at $72{ }^{\circ} \mathrm{C}$ for $1 \mathrm{~s}$. The reaction was preceded by a primary denaturation step at $95{ }^{\circ} \mathrm{C}$ for $15 \mathrm{~s}$.

PCR products were separated on $1 \% \mathrm{w} / \mathrm{v}$ agarose gels stained with ethidium bromide and were purified using the PCR and DNA Fragment Purification kit (Dongsheng Biotech, DNA purity 1.7-1.9). Sanger DNA sequencing by capillary electrophoresis was applied to detect the genotypes for VEGF-2578C/A (rs699947), VEGF-1154G/A (rs1570360) and VEGF-634G/C (rs2010963) SNPs (3130 genetic analyzer, Applied Biosystems $\left.{ }^{\circledR}\right)[33,34]$.

Model development

The nonlinear mixed-effects modeling software NONMEM $^{\circledR} 7.3$ (Icon Development Solutions, Ellicott City, MD, USA) was used in the data analysis. All population parameter estimates were obtained with the first-order conditional estimation method with interaction. The graphical representation of the data and model diagnostics was performed with the software tool Xpose (version 4.3.5) [35]. The PsN toolkit (version 4.2.0) was used for the implementation of computer-intensive statistical methods (stepwise covariate model building and randomization test) [36]. Typical concentration-time profiles for total bevacizumab, total and free $\mathrm{VEGF}_{165}$ were generated using Berkeley Madonna (version 8.3.18, Kagi Shareware, Berkeley, CA, USA).

Inter-individual variability in model parameters was tested assuming a log-normal distribution described by an exponential model (Eq. 1), 
$P_{i}=P_{p} \cdot \exp \left(\eta_{i, p}\right)$

where $P_{i}$ represents the parameter estimate for the $i$ th individual, $P_{p}$ the typical parameter estimate in the population and $\eta_{i, p}$ the random variable for the $i$ th individual from a normal distribution with a mean of zero and an estimated variance of $\omega^{2}$. Proportional, additive and combined error models on normal scale or additive and combined error models on log-transformed scale were explored to describe the unexplained residual variability. The magnitude of inter-individual and residual variability was expressed as $\mathrm{CV} \%$.

The likelihood ratio test was used to assess whether the difference in the objective function $(\Delta \mathrm{OFV})$ between different (sub)models (assumed to be $\chi^{2}$ distributed) was statistically significant. The evaluation of basic goodnessof-fit plots, shrinkage [37] and parameter uncertainty were also taken into account for model discrimination.

Plots of individual empirical Bayes (post hoc) estimates of the PK and VEGF-related parameters versus covariates were explored for the identification of potential parameter-covariate relationships. The choice of the covariate model was based on a stepwise covariate model procedure, a PsN feature that implements forward selection and backward elimination of covariates to a model according to statistical criteria [36]. A decrease in OFV of 3.84 points for forward inclusion $(p<0.05$, for one parameter difference) and an increase of 6.64 points for backward deletion $(p<0.01$, for one parameter difference) were considered significant.

The following covariates were tested for significance on model parameters: actual body weight, age, gender and VEGF-A SNPs $(-2578 \mathrm{C} / \mathrm{A},-1154 \mathrm{G} / \mathrm{A}$ and $-634 \mathrm{G} / \mathrm{C})$. Linear and power parameterizations were considered for the continuous covariates (Eqs. 2, 3, respectively), whereas the categorical covariates were tested in linear equations (Eq. 4). The equations are shown below:

$P_{i}=P_{p} \cdot\left[1+\theta \cdot\left(\operatorname{Cov}-\operatorname{Cov}_{\text {med. }}\right)\right] \cdot \exp \left(\eta_{i, p}\right)$

$P_{i}=P_{p} \cdot\left(\frac{\operatorname{Cov}}{\operatorname{Cov}_{\text {med. }}}\right)^{k} \cdot \exp \left(\eta_{i, p}\right)$

$P_{i}=P_{p} \cdot(1+\theta \cdot \operatorname{Cov}) \cdot \exp \left(\eta_{i, p}\right)$

In these equations, Cov represents the covariate and $\operatorname{Cov}_{\text {med. }}$ the median value of the covariate in the study population. $\theta$ is the fractional change in the population parameter for an individual with a covariate value different from the median value (Eq. 2) or a reference value (Eq. 4). $k$ stands for the exponential scaling factor and in case of body weight, it was either estimated or fixed to a certain value (0.75 for clearance and 1 for volume parameters) [38].
A randomization test was performed to calculate the probability of identifying falsely significant covariates [39]. A total of 200 new datasets were generated by shuffling 200 times the sequence of the covariate values between individuals in the randomization column. A base model (without a parameter-covariate relationship) was fitted to the original dataset. Then, a full model (with a parameter-covariate relationship) was fitted to the original and the randomized datasets, and the $\triangle \mathrm{OFV}$ s from the base model were computed. Based on the distribution of the difference in OFV between the two models for each of the 200 datasets, the actual drop in OFV required to reach the $5 \%$ significance level was calculated.

Data analysis was performed in two separate steps. As a first step, only total bevacizumab concentration-time (PK) data were included in the analysis and a population PK model for bevacizumab was developed to gain information on the PK profile of the drug in the current study population. A previously published model by Lu et al. [26], which describes bevacizumab data in a similar study population in terms of demographic and study characteristics, was used as a reference to evaluate whether the present PK data are in reasonable agreement with the previous observations. In the second step of data analysis, a simultaneous fit of total bevacizumab and free $\mathrm{VEGF}_{165}$ concentration-time data was performed to develop a binding model for bevacizumab using a TMDD modeling approach. The simultaneous data analysis allowed for the exploration of the influence of $\mathrm{VEGF}_{165}$ on bevacizumab disposition and eliminated the effect of potential shrinkage in PK parameters on the estimation of VEGF-related parameters.

PK model

Total bevacizumab concentrations in serum were expressed in $\mathrm{mg} / \mathrm{L}$.

In the first step of data analysis, one- and two-compartment models with first-order elimination were investigated to describe bevacizumab concentration changes over time.

The effect of body weight, age and gender was tested on all PK parameters taking into account prior knowledge on the highly influential covariates $[25,26]$ and the clinical relevance of the available covariates. In addition, the influence of VEGF-A SNPs $(-2578 \mathrm{C} / \mathrm{A},-1154 \mathrm{G} / \mathrm{A}$ and $-634 \mathrm{G} / \mathrm{C}$ ) was only explored on clearance parameters, as these polymorphisms are associated with VEGF production or promoter activity [12-14].

Inter-individual variability was investigated in all model parameters, and an error model was developed to describe the unexplained residual variability.

All parameters of the developed PK model were estimated, and the PK parameter estimates reported by $\mathrm{Lu}$ et al. [26] were only used as initial estimates. The ability of 
the proposed model to yield plausible parameter estimates was tested by comparing them with the parameter estimates obtained by the model of Lu et al., which predicts the concentration-time profile of bevacizumab in a larger study population.

\section{TMDD (binding) model}

Total bevacizumab and free $\mathrm{VEGF}_{165}$ concentrations in serum were expressed in $\mathrm{nM}$. Modeling was performed on log-transformed data.

In the second step of data analysis, the structural and covariate form of the developed PK model for bevacizumab was linked with a QSS TMDD model to simultaneously describe total bevacizumab and free $\mathrm{VEGF}_{165}$ concentration-time profiles.

The QSS equations [22-24] for the concentrations of total bevacizumab $\left(C_{\mathrm{tot}}\right)$, total $\mathrm{VEGF}_{165}\left(R_{\mathrm{tot}}\right)$, free bevacizumab $(C)$, bevacizumab-VEGF 165 complex $(R C)$ and free $\operatorname{VEGF}_{165}(R)$ are shown below:

$\frac{\mathrm{d} C_{\mathrm{tot}}}{\mathrm{d} t}=\frac{\operatorname{In}(t)}{V_{1}}-\left(\frac{\mathrm{CL}}{V_{1}}+\frac{Q}{V_{1}}\right) \cdot C-\frac{R_{\mathrm{tot}} \frac{\mathrm{CL}_{R C}}{V_{1}} C}{K_{\mathrm{ss}}+C}+\frac{\frac{Q}{V_{2}} A_{2}}{V_{1}}$

$\frac{\mathrm{d} R_{\mathrm{tot}}}{\mathrm{d} t}=k_{\mathrm{in}}-k_{\mathrm{out}} \cdot R_{\mathrm{tot}}-\left(\frac{\mathrm{CL}_{R C}}{V_{1}}-k_{\mathrm{out}}\right) \frac{R_{\mathrm{tot}} \cdot C}{K_{\mathrm{ss}}+C}$

$C=\frac{1}{2}\left[\left(C_{\mathrm{tot}}-R_{\mathrm{tot}}-K_{\mathrm{ss}}\right)+\sqrt{\left(C_{\mathrm{tot}}-R_{\mathrm{tot}}-K_{\mathrm{ss}}\right)^{2}+4 \cdot K_{\mathrm{ss}} \cdot C_{\mathrm{tot}}}\right]$

$R C=\frac{R_{\mathrm{tot}} \cdot C}{K_{\mathrm{ss}}+C}$

$R=R_{\mathrm{tot}}-R C$

In these equations, $\operatorname{In}(\mathrm{t})$ is the infusion rate; $A_{2}$ is the amount of the free bevacizumab in the peripheral compartment; $V_{1}$ and $V_{2}$ are the volumes of distribution of the central and peripheral compartment, respectively; $\mathrm{CL}$ is the elimination clearance of free bevacizumab; $\mathrm{CL}_{R C}$ is the elimination clearance of the bevacizumabVEGF $_{165}$ complex; $Q$ is the intercompartmental clearance. $k_{\text {in }}$ and $k_{\text {out }}$ are the production (zero-order) and elimination (first-order) rate constants of free $\mathrm{VEGF}_{165}$. $K_{\mathrm{ss}}$ is the steady-state constant that defines the QSS among free bevacizumab, free $\mathrm{VEGF}_{165}$ and bevacizumab-VEGF $_{165}$ complex and is described by the following equation:

$K_{\mathrm{ss}}=\frac{k_{\mathrm{int}}+k_{\mathrm{off}}}{k_{\mathrm{on}}}$ $k_{\text {int }}, k_{\text {off }}$ and $k_{\text {on }}$ are the elimination (first-order), dissociation (first-order) and binding (second-order) rate constants of the bevacizumab-VEGF ${ }_{165}$ complex.

VEGF-A SNPs $(-2578 \mathrm{C} / \mathrm{A},-1154 \mathrm{G} / \mathrm{A}$ and $-634 \mathrm{G} / \mathrm{C})$ were only tested for significance on VEGF-related parameters.

Inter-individual variability was investigated in all PK and VEGF-related parameters, and an error model was developed to describe the unexplained residual variability.

All parameters of the developed TMDD model were estimated, and the population PK parameter estimates from the first step of data analysis were only used as initial estimates.

\section{Model evaluation}

The predictive performance of the developed models for bevacizumab (PK and TMDD model) was evaluated using prediction-corrected visual predictive checks (pcVPCs) [40, 41]. A total of 1000 simulated datasets were generated, and the $95 \%$ confidence intervals for the median, 10th and 90th percentiles of the simulated data were compared to the median, 10th and 90th percentiles of the observed data. The uncertainty in model parameters was expressed by the relative standard error (RSE) obtained by the covariance step in NONMEM.

\section{Results}

Patients and samples

The current analysis was based on 86 total bevacizumab and 93 free VEGF $_{165}$ serum concentrations collected from 19 adult patients with stage IV CRC. None of the measured concentrations was below the limit of quantification. Blood samples (median 4, 2-10 per patient) were collected before bevacizumab administration and immediately after the end of infusion.

Ten patients received bevacizumab in combination with FOLFIRI or FOLFOX treatment, whereas nine patients were administered bevacizumab together with CAPIRI treatment. Only three patients switched over to bevacizumab monotherapy (one patient from each treatment group). The patient on BEV-CAPIRI treatment had received nine cycles of bevacizumab before changing to bevacizumab monotherapy.

The study population was predominately male (58\%), with a median age of 60 years and median body weight of $70 \mathrm{~kg}$. The most frequent genotypes were VEGF-2578CA (58 \%) for VEGF-2578C/A, VEGF-1154GG (53\%) for VEGF-1154G/A and VEGF-634GG (74 \%) for VEGF634G/C SNPs. 
Table 1 Patient and study characteristics

\begin{tabular}{|c|c|}
\hline Characteristic & Value \\
\hline Dose (mg/kg) & 5 or 10 biw, 7.5 tiw \\
\hline Duration of infusion (min) & $85(40-90)$ \\
\hline \multicolumn{2}{|l|}{ Treatment period (days) } \\
\hline BEV-FOLFIRI or BEV-FOLFOX treatment & $127.5(27-348)$ \\
\hline BEV-CAPIRI treatment & $174(71-251)$ \\
\hline Actual body weight (kg) & $70(50-94)$ \\
\hline Age (years) & $60(37-73)$ \\
\hline Gender (M/F) & $11 / 8(58 / 42 \%)$ \\
\hline \multicolumn{2}{|l|}{ Total bevacizumab concentrations (mg/L) } \\
\hline Pre-dose concentrations & $83.6(13.5-172)$ \\
\hline Post-dose concentrations & $203.8(71.1-412)$ \\
\hline \multicolumn{2}{|l|}{ Free VEGF $_{165}$ concentrations $(\mathrm{ng} / \mathrm{L})$} \\
\hline Pre-dose concentrations & $187.7(52.3-451.6)$ \\
\hline Post-dose concentrations & $76.5(16.9-371.6)$ \\
\hline \multicolumn{2}{|l|}{ Bevacizumab samples/cycle } \\
\hline BEV-FOLFIRI or BEV-FOLFOX treatment & $2(1-8)$ \\
\hline BEV-CAPIRI treatment & $4(1-10)$ \\
\hline \multicolumn{2}{|l|}{ VEGF $_{165}$ samples/cycle } \\
\hline BEV-FOLFIRI or BEV-FOLFOX treatment & $2(1-8)$ \\
\hline BEV-CAPIRI treatment & $3.5(1-9)$ \\
\hline $\mathrm{VEGF}_{2578 \mathrm{C} / \mathrm{A}}\left(\mathrm{CC}^{\mathrm{a}} / \mathrm{AA}^{\mathrm{b}} / \mathrm{CA}^{\mathrm{c}}\right)$ & $2 / 6 / 11(10 / 32 / 58 \%)$ \\
\hline $\operatorname{VEGF}_{1154 \mathrm{G} / \mathrm{A}}\left(\mathrm{GG}^{\mathrm{a}} / \mathrm{AA}^{\mathrm{b}} / \mathrm{GA}^{\mathrm{c}}\right)$ & $10 / 2 / 7(53 / 10 / 37 \%)$ \\
\hline $\operatorname{VEGF}_{634 \mathrm{G} / \mathrm{C}}\left(\mathrm{GG}^{\mathrm{a}} / \mathrm{CC}^{\mathrm{b}} / \mathrm{GC}^{\mathrm{c}}\right)$ & $14 / 1 / 4(74 / 5 / 21 \%)$ \\
\hline
\end{tabular}

Values are either expressed as median (range) or absolute values (\%) biw every 2 weeks, tiw every 3 weeks, $M$ male, $F$ female

${ }^{\mathrm{a}}$ Wild type; ${ }^{\mathrm{b}}$ Homozygous; ${ }^{\mathrm{c}}$ Heterozygous

A summary of the patient and study characteristics is shown in Table 1.

\section{VEGF assay}

The ability of the Quantikine ${ }^{\circledR}$ human VEGF ELISA assay [32] to detect free or total $\mathrm{VEGF}_{165}$ in the presence of bevacizumab was tested in vitro by mixing $\mathrm{VEGF}_{165}$ standards (1000 and $250 \mathrm{ng} / \mathrm{L}$ ) with increasing bevacizumab concentrations. The measured $\mathrm{VEGF}_{165}$ concentrations were equal to zero when bevacizumab was in excess confirming that only free $\mathrm{VEGF}_{165}$ can be detected by this VEGF ELISA assay, otherwise the measured $\mathrm{VEGF}_{165}$ concentrations should be identical to the concentrations of $\mathrm{VEGF}_{165}$ standards (1000 and $250 \mathrm{ng} / \mathrm{L})$. Mean (RSE \%) VEGF 165 concentrations for $\mathrm{VEGF}_{165}$ to bevacizumab 1:0, 1:0.1, 1:1 and 1:1000 molar ratios are shown in Table 2.

PK model

A two-compartment model with first-order elimination was found to describe bevacizumab concentration
Table 2 In vitro VEGF $_{165}$ concentrations after addition of bevacizumab

\begin{tabular}{llc}
\hline $\begin{array}{l}\text { VEGF }_{165} \\
\text { standards (ng/L) }\end{array}$ & $\begin{array}{l}\text { VEGF }_{165:} \text { bevacizumab } \\
\text { molar ratio }\end{array}$ & $\begin{array}{l}\text { VEGF }_{165} \\
\text { concentrations }(\mathrm{ng} / \mathrm{L})\end{array}$ \\
\hline 1000 & $1: 0$ & $1120.2(2.67)$ \\
& $1: 0.1$ & $448.5(1.66)$ \\
& $1: 1$ & $452.2(0.83)$ \\
250 & $1: 1000$ & $0(0)$ \\
& $1: 0$ & $172.4(4.33)$ \\
& $1: 0.1$ & $79.1(4.72)$ \\
& $1: 1$ & $79.1(4.72)$ \\
& $1: 1000$ & $0(0)$ \\
\hline
\end{tabular}

VEGF $_{165}$ concentrations are expressed as average (RSE \%)

changes over time better than a one-compartment model $(\triangle \mathrm{OFV}=-18.9)$. The selection of the final structural model was further supported by basic goodness-of-fit plots and a previously published model for bevacizumab [26]. Goodness-of-fit plots showed no apparent bias in residuals over time or across population-predicted bevacizumab concentrations (Supplemental Fig. 2).

$V_{1}$ and $V_{2}$ were found to be 3.14 and $2.63 \mathrm{~L}$, respectively. Bevacizumab CL was $0.17 \mathrm{~L} /$ day, and $Q$ was $0.36 \mathrm{~L} /$ day.

Inter-individual variability could be identified for CL $(23 \%)$ and $V_{1}(15 \%)$. The residual variability was explained by a proportional error model (24\%).

In the stepwise covariate analysis, the effect of several covariates (body weight, age, gender and VEGF-A SNPs) was explored on the PK parameters. None of the available covariates provided an increase of 6.64 points in OFV for backward deletion ( $p<0.01$, for one parameter difference). Body weight was incorporated with an allometric function in all clearance and volume parameters to ensure model stability considering the strong biological covariate relationship [38, 42] and prior information from studies in patients with solid tumors receiving bevacizumab [25, 26].

Model parameters could be estimated with acceptable precision (RSE $<25 \%$ ) except for $V_{2}$ (RSE $50 \%$ ), $Q$ (RSE $146 \%$ ) and inter-individual variability in $V_{1}$ (RSE $38 \%$ ). The parameter estimates from the PK model and their RSEs are represented in Table 3.

TMDD (binding) model

A QSS TMDD model was applied to explore the in vivo interaction between bevacizumab and its soluble target, VEGF $_{165}$. In this model, free bevacizumab is eliminated from the central compartment through two pathways: (1) degradation (linear CL) and (2) binding to $\mathrm{VEGF}_{165}$ and subsequent degradation of the bevacizumab-VEGF 165 
Table 3 Population parameter estimates from the PK model (bevacizumab analyzed alone) and TMDD model (bevacizumab and VEGF 165 analyzed simultaneously)

\begin{tabular}{|c|c|c|c|c|}
\hline \multirow[t]{2}{*}{ Parameter } & \multicolumn{2}{|l|}{ PK model } & \multicolumn{2}{|l|}{ TMDD model } \\
\hline & Typical value (RSE \%) & IIV, CV \% (RSE \%) & Typical value (RSE \%) & IIV, CV \% (RSE \%) \\
\hline CL (L/day) & $0.17(11)$ & $23(23)$ & $0.18(6)$ & $20(25)$ \\
\hline$V_{1}(\mathrm{~L})$ & $3.14(7)$ & $15(38)$ & $3.23(7)$ & $22(36)$ \\
\hline$Q$ (L/day) & $0.36(146)$ & & $1.38(19)$ & \\
\hline$V_{2}(\mathrm{~L})$ & $2.63(50)$ & & $3.1(18)$ & \\
\hline $\mathrm{BM}_{0}(\mathrm{ng} / \mathrm{L})$ & & & $212(8)$ & $33(23)$ \\
\hline$k_{\text {out }}(/$ day $)$ & & & $0.401(14)$ & \\
\hline$K_{\mathrm{ss}}(\mathrm{nM})$ & & & $267(22)$ & \\
\hline Prop. error ${ }_{\mathrm{bev}}(\%)$ & $24(13)$ & & $28(15)$ & \\
\hline Prop. error ${ }_{\mathrm{VEGF165}}(\%)$ & & & $32(14)$ & \\
\hline
\end{tabular}

$C L$ clearance, $V_{l}$ volume of central compartment, $Q$ intercompartmental clearance, $V_{2}$ volume of peripheral compartment, $B M_{0}$ baseline $\mathrm{VEGF}_{165}, k_{\text {out }}$ elimination rate constant of $\mathrm{VEGF}_{165}, K_{s s}$ steady-state constant, $R S E$ relative standard error, $I I V$ inter-individual variability, $C V$ coefficient of variation, bev bevacizumab

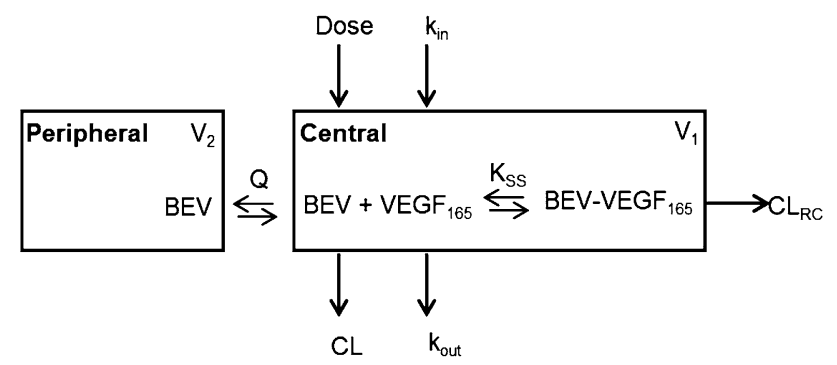

Fig. 1 Structure of the binding model for bevacizumab-VEGF 165 interaction. The approximation $\mathrm{CL}_{R C}=\mathrm{CL}$ was used for purposes of model fitting

complex (linear $\mathrm{CL}_{R C}$ ). The structure of the binding model is shown in Fig. 1.

The developed model adequately described the time course of total bevacizumab and free $\mathrm{VEGF}_{165}$ serum concentrations in the current study population. The estimated $\mathrm{CL}$ of free bevacizumab was $0.18 \mathrm{~L} /$ day, and $Q$ was $1.38 \mathrm{~L} /$ day. The information in the present data was not sufficient to allow for a separate estimation of the elimination clearance of the bevacizumab-VEGF ${ }_{165}$ complex, which was therefore set equal to the $\mathrm{CL}$ of the free bevacizumab $\left(\mathrm{CL}_{R C}=\mathrm{CL}\right.$ ). Baseline $\mathrm{VEGF}_{165}$ (free $\mathrm{VEGF}_{165}$ at time 0, $\mathrm{BM}_{0}$ ) was $0.0053 \mathrm{nM}$ corresponding to $212 \mathrm{ng} / \mathrm{L}$ (assuming a 1:1 molecular interaction), and $k_{\text {out }}$ was 0.401 day $^{-1}$. $K_{\mathrm{ss}}$ was found to be $267 \mathrm{nM}$. $k_{\mathrm{in}}$, which is defined as the typical value of $\mathrm{BM}_{0}$ times the typical value of $k_{\text {out }}$ $\left(\mathrm{BM}_{0, P} \times k_{\text {out }, P}\right)$, was $85 \mathrm{ng} / \mathrm{L} /$ day.

Inter-individual variability was assigned to the following parameters: $\mathrm{CL}, V_{1}$ and $\mathrm{BM}_{0}$. The residual variability in total bevacizumab and free $\mathrm{VEGF}_{165}$ concentrations was explained by an additive error model on log-transformed data.
No direct relationship between VEGF-A SNPs and $\mathrm{BM}_{0}, k_{\text {out }}$ or $K_{\text {ss }}$ was identified. However, patients with VEGF-2578AA (homozygous), VEGF-634CC (homozygous) and VEGF-634GC (heterozygous) genotypes seem to have a larger $K_{\mathrm{ss}}$. The effect of VEGF-634G/C polymorphism on $K_{\mathrm{ss}}$ becomes significant $(\Delta \mathrm{OFV}=-8.6)$ only after the inclusion of VEGF-2578C/A polymorphism in $K_{\mathrm{ss}}(\Delta \mathrm{OFV}=-6)$. A plot of $\triangle \mathrm{OFV}$ versus individuals indicated that one specific individual was driving the relationship between VEGF-2578C/A and $K_{\text {ss }}$ (data not shown). A randomization test was then performed to explore whether the inclusion of these covariate relationships was falsely significant. It was concluded that the observed trends between VEGF-2578C/A and $K_{\mathrm{ss}}$ or VEGF-634G/C and $K_{\text {ss }}$ did not reach statistical significance as the required drop in OFV after the inclusion of VEGF-2578C/A or VEGF-634G/C was 6.8 points or 6.7 points, respectively ( $p<0.05$, forward inclusion of one parameter).

All the PK and VEGF-related parameter estimates from the binding model and their RSEs are represented in Table 3. The RSEs of the fixed and random effects remained well below $25 \%$ except for the inter-individual variability in $V_{1}$ (RSE $36 \%$ ), indicating that the parameters could be estimated with acceptable precision. A low $\varepsilon$-shrinkage $(10 \%)$ suggests that the model diagnostics are reliable. However, $\eta$-shrinkage was relatively high for $V_{1}$ (34\%) but $<22 \%$ for CL and $\mathrm{BM}_{0}$.

Evaluation of the TMDD (binding) model

The TMDD model had a good predictive performance, as expressed by the results of the VPC depicted in Fig. 2. It can adequately describe the time course of total 
Fig. 2 Prediction-corrected visual predictive checks of the binding model based on 1000 simulations (panel a total bevacizumab, panel $\mathbf{b}$ free $\mathrm{VEGF}_{165}$ ). Median (solid line), 10th and 90th percentiles (dashed lines) of the observed data (circles) are compared to the $95 \%$ confidence intervals (shaded areas) for the median, 10th and 90th percentiles of the simulated data
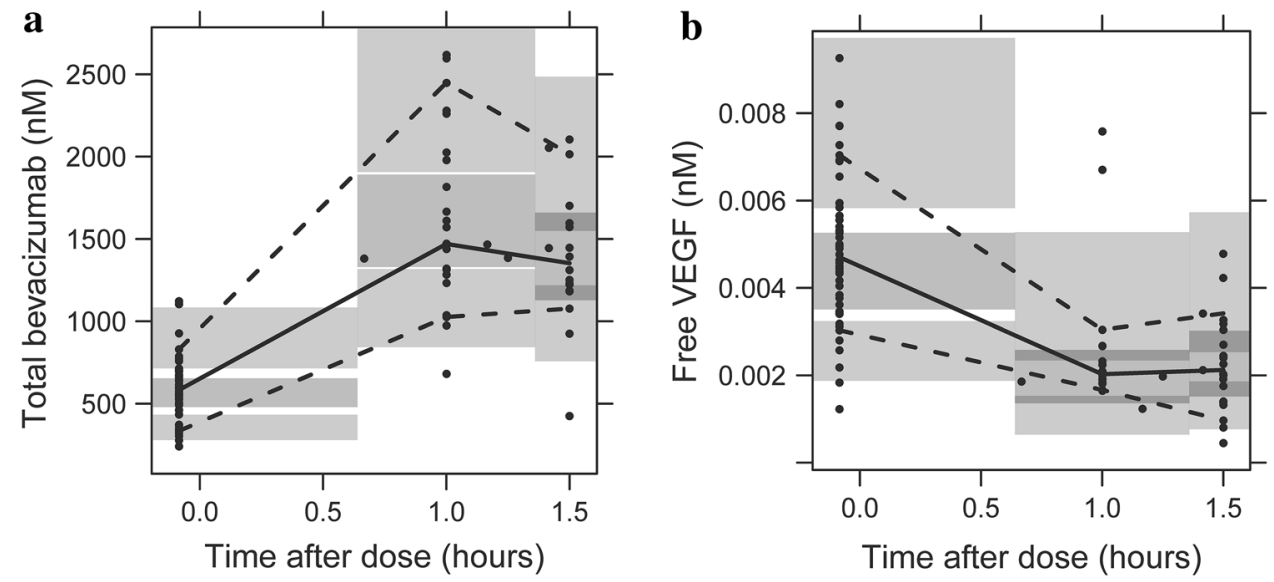

bevacizumab and free VEGF $_{165}$ concentrations as well as the observed variability in the study population.

\section{Simulations}

The concentration-time profiles of total bevacizumab, total and free $\mathrm{VEGF}_{165}$ for a typical patient of $70 \mathrm{~kg}$ receiving bevacizumab either at a dose of $5 \mathrm{mg} / \mathrm{kg}$ every 2 weeks or $7.5 \mathrm{mg} / \mathrm{kg}$ every 3 weeks (the most frequent dosing regimens in the study population) are shown in Fig. 3.

A significant drop in the free $\mathrm{VEGF}_{165}$ levels was observed upon administration of the first dose $(73 \%$ for the lower dose and $80 \%$ for the higher dose) followed by a less pronounced decline on subsequent doses attributed to the reversible formation and accumulation of bevacizumab-VEGF $_{165}$ complexes (Fig. 3a, b, respectively). The total $\mathrm{VEGF}_{165}$ concentrations increased over time, in both dosing regimens, up to a level where no more complexes could be formed. The extent of total $\mathrm{VEGF}_{165}$ accumulation seemed less at the higher dose compared to the lower dose, while the decline in free $\mathrm{VEGF}_{165}$ levels was slightly increased (54\% compared to $42 \%$ for the lower dose, Fig. 3c).

\section{Discussion}

In this study, a binding model for bevacizumab was developed to characterize its PK behavior, to describe its binding properties to $\mathrm{VEGF}_{165}$ and to assess the influence of relevant covariates (e.g., genetics) on the relationship between bevacizumab and VEGF $_{165}$ in adult patients with stage IV CRC. Sparse bevacizumab and VEGF $_{165}$ data were collected during routine clinical practice from 19 adult patients following bevacizumab treatment in combination with chemotherapy (FOLFIRI, FOLFOX or CAPIRI). Data analysis was performed in two distinct steps using nonlinear mixed-effects modeling.
In the first step of data analysis, a PK model for bevacizumab was developed and the model-predicted profiles were compared to the profiles reported by $\mathrm{Lu}$ et al. [26] to evaluate whether the data collected in the current study were in accordance with previous observations. Although the two-compartment model did not provide a pronounced improvement of the model fit over a one-compartment model, it was found to support the previously reported model structure and parameters and was therefore selected.

In the second step of data analysis, a TMDD model using the QSS approximation [22-24] was developed to characterize the in vivo bevacizumab-VEGF 165 interaction based on a simultaneous analysis of total bevacizumab and free $\mathrm{VEGF}_{165}$ serum concentrations. The simultaneous data fitting allowed for the evaluation of the effect of bevacizumab on the reduction in free $\mathrm{VEGF}_{165}$ levels and shed light on the in vivo binding affinity of bevacizumab to $\mathrm{VEGF}_{165}$ as most of the current data come from in vitro biological studies [43, 44]. Furthermore, it provided some valuable information on the PK properties of both bevacizumab and $\mathrm{VEGF}_{165}$ in patients with cancer.

The TMDD model could adequately describe total bevacizumab and free VEGF $_{165}$ profiles observed during bevacizumab treatment. The simultaneous data analysis allowed for a higher precision in the estimation of PK parameters compared to the analysis of bevacizumab data alone, indicating that $\mathrm{VEGF}_{165}$ concentrations may carry information on bevacizumab disposition. In this model, the typical volume of the central and peripheral compartment was similar to the values previously reported by $\mathrm{Lu}$ et al. [26] and Gaudreault et al. [45]. Bevacizumab clearance was found to be consistent with that observed by $\mathrm{Lu}$ et al. (0.21 L/day) [26] and Gordon et al. (0.19-0.36 L/ day for a typical patient of $70 \mathrm{~kg}$ ) [46]. The estimated free VEGF $_{165}$ levels at baseline as well as the elimination rate constant, $k_{\text {out }}$, of free $\mathrm{VEGF}_{165}$ were also in line with published values in patients with advanced cancer [47, 48]. However, a much larger $K_{\mathrm{ss}}(267 \mathrm{nM})$ than the in vitro 
Fig. 3 Model-predicted concentrations of total bevacizumab, total and free VEGF $_{165}$ for a typical patient of $70 \mathrm{~kg}$. Panels a, b show the total bevacizumab and free VEGF $_{165}$ concentration profiles at doses of 5 and $7.5 \mathrm{mg} / \mathrm{kg}$, respectively. Panel $\mathbf{c}$ depicts the total $\mathrm{VEGF}_{165}$ profiles over time for the two dosing regimens
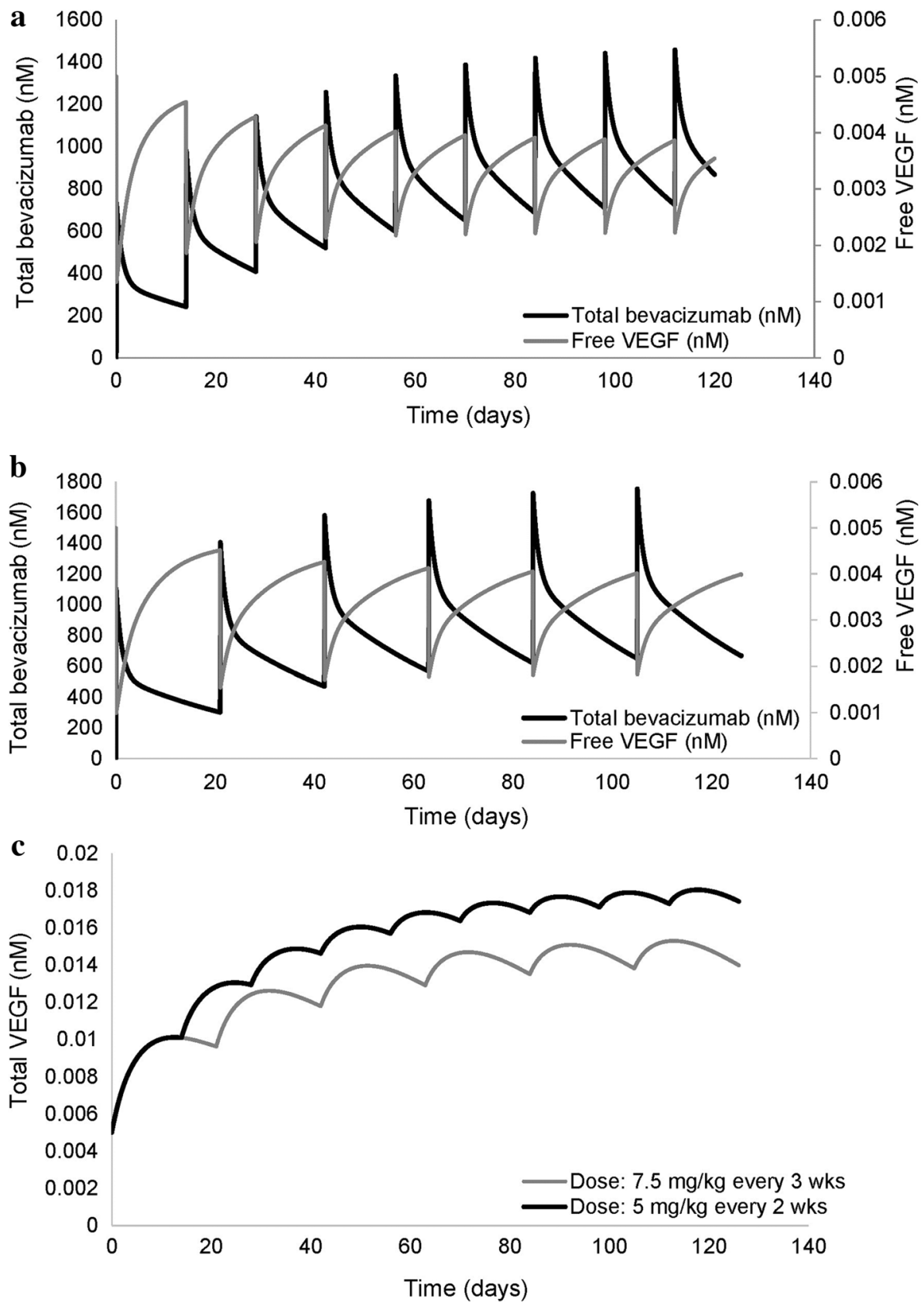

equilibrium dissociation constant (KD) of bevacizumab for VEGF-A (1.8-20 nM) [1, 44] was observed. This finding is in agreement with the QSS approximation that often predicts a greater $K_{\mathrm{ss}}$ value in vivo than the in vitro KD $[21,23]$.

Model-based simulations (Fig. 3) revealed a significant drop $(73-80 \%)$ in the free serum VEGF $_{165}$ concentrations upon administration of the first dose of bevacizumab, which reaches a pseudo-steady-state after multiple doses. A similar behavior was suggested by Stefanini et al. [49] in case bevacizumab is confined to the blood compartment. They mentioned a decline of $87 \%$ in free serum $\mathrm{VEGF}_{165}$ levels after the first dose of bevacizumab. Model predictions regarding the effect of bevacizumab on the reduction in free $\mathrm{VEGF}_{165}$ levels in serum are of particular importance as they could indicate when dose adjustments are needed to achieve sufficient VEGF-A blockade and thus optimal anti-angiogenic activity of bevacizumab. Increases in total serum VEGF $_{165}$ concentrations were also noticed, as in previous studies by Gordon et al. [46] and Stefanini et al. [49]. This increase in total VEGF $_{165}$ could be a result of bevacizumab-VEGF ${ }_{165}$ complex dissociation, a decrease in $\mathrm{VEGF}_{165}$ clearance caused by the complexation process [50] or a constant production rate of $\mathrm{VEGF}_{165}$. 
The effect of demographic characteristics and genetics on the PK and VEGF-related parameters in the target population was explored. Among all covariates tested, none was found to be significant according to the predefined statistical criteria. The difficulty in identifying any significant covariate effects could be attributed to the sparse sampling schedule and the small size of the study population. Thus, the developed TMDD (binding) model did not include any covariates except for body weight in all clearance and volume parameters to ensure model stability, considering the strong biological prior $[38,42]$ and the outcome of previous analyses [25, 26]. Despite the reported gender differences in bevacizumab clearance and volume of distribution [26, 45], the gender effect was not retained in the final model because gender and patient body weight are often strongly correlated, and thus, the inclusion of body weight is usually sufficient to completely describe the gender influence. The effect of serum albumin and alkaline phospatase on clearance could not be assessed in the current study since data were not available for all patients. The influence of co-administered chemotherapy (FOLFIRI, FOLFOX, CAPIRI) was not tested on bevacizumab PK, as more information would be needed to unravel potential drug-drug interactions. Nevertheless, published data indicate no significant PK interactions between bevacizumab and other antineoplastic agents $[1,26]$.

Interestingly, a larger $K_{\mathrm{ss}}$ value was observed for patients with VEGF-2578AA, VEGF-634CC and VEGF-634GC genotypes. It is noteworthy that when the covariate effect of VEGF-2578C/A or VEGF-634G/C polymorphism was added in $K_{\mathrm{ss}}$, none of the model-predicted parameters was substantially affected except for the value of $K_{\mathrm{ss}}$, which was reduced from 267 to 221 or $176 \mathrm{nM}$, respectively. This finding indicates that VEGF-2578C/A and VEGF-634G/C polymorphisms might be predictive for the binding affinity of bevacizumab to $\mathrm{VEGF}_{165}$, as $K_{\mathrm{ss}}$ and affinity of the drug to its molecular target are correlated (Eq. 10). Larger cohort studies in bevacizumab-treated patients are needed though, to confirm these results and to assess the potential role of VEGF-2578C/A and VEGF-634G/C polymorphisms in guiding patient and dose selection.

Some limitations of our study should be also addressed. The elimination clearance of the bevacizumab-VEGF ${ }_{165}$ complex could not be estimated and it was set equal to the clearance of the free bevacizumab, allowing for a simultaneous model fit of total bevacizumab and free $\mathrm{VEGF}_{165}$ serum concentrations. Free bevacizumab $(\sim 150 \mathrm{kDa})$ is known to undergo proteolytic catabolism mediated by the neonatal $\mathrm{Fc}$ receptor $(\mathrm{FcRn})$, which contributes to the slow elimination of the drug from the systemic circulation [12]. It is likely that the bevacizumab-VEGF ${ }_{165}$ complex (assuming a 1:1 molecular interaction), which has a similar molecular weight with bevacizumab $(\sim 190 \mathrm{kDa})$ and is salvaged from degradation through binding of its Fc moiety to FcRn, exhibits a similar elimination rate. This hypothesis is further supported by Hsei et al. [50], who observed no statistically significant difference between the clearance of the free bevacizumab and the bevacizumab-VEGF ${ }_{165}$ complex in rats. In the current study, the free drug and the complex were also assumed to be distributed in the same space, given that such large molecules would extravasate relatively slow. However, Hsei et al. [50] demonstrated a small, though significant change (20-25\%) in the volumes of distribution, implying that free bevacizumab and bevacizumab-VEGF ${ }_{165}$ complex might not be distributed exactly in the same space. A future study in humans, which would provide information on the concentration-time profile of the bevacizumab-VEGF ${ }_{165}$ complex, could therefore shed more light on the disposition properties of the complex and detect potential interspecies differences. Moreover, it was hypothesized that only one type of complex can be formed through monomeric binding (bevacizumab bound to one molecule of $\mathrm{VEGF}_{165}$ ). The possibility of formation of other complexes through multimeric binding (e.g., bevacizumab bound to two molecules of $\mathrm{VEGF}_{165}$, VEGF $_{165}$ bound to two molecules of bevacizumab or other immune complexes) was ignored, as the bevacizumab bioassay could only detect the complex formed between bevacizumab and one molecule of $\mathrm{VEGF}_{165}$. The quantification of higher-order immune complexes is considered extremely difficult due to their rapid elimination from the systemic circulation [51]. Their assessment, though, could be proved valuable in reflecting an additional clearance pathway for bevacizumab. The effect of platelets on taking up VEGF and bevacizumab [52] was not included in the current model but it would be useful to be added when more data become available, as it could provide information on the fluctuation of serum VEGF levels during bevacizumab treatment.

\section{Conclusion}

In conclusion, the developed binding model adequately characterizes the PK of bevacizumab and the relationship between bevacizumab and $\mathrm{VEGF}_{165}$ in adult patients with stage IV CRC receiving bevacizumab treatment in combination with chemotherapy. To the best of our knowledge, this is the first time a TMDD model was applied to characterize the in vivo interaction of bevacizumab with its soluble ligand, VEGF ${ }_{165}$. Although no significant effect of VEGF-A polymorphisms on the relationship between bevacizumab and $\mathrm{VEGF}_{165}$ was identified, correlations between the binding affinity of bevacizumab to $\mathrm{VEGF}_{165}$ and the VEGF2578C/A and VEGF-634G/C SNPs were noticed. This model could serve as a basis for further studies to elucidate 
the role of VEGF-A polymorphisms and serum VEGF levels in treatment response and to support dose rationale for bevacizumab when combined with chemotherapy.

Acknowledgments Panoilia E. was financially supported in part by funds of the European Program "Education and Lifelong Learning" of NSRF 2007-2013 through IKY Scholarships, Greece. Schindler E. was financially supported by the Swedish Cancer Society, Sweden. We thank Kiosse G., Koukourikou I., Panou I., Patinioti I., Vicha A. for their assistance in the data collection, Dedoglou A., Paizi A. for performing the genotype analysis and Brekkan A. for his contribution to model development.

Conflict of interest Kalofonos H.P. serves in a consultant/advisory role to Amgen, Roche, Novartis, Pfizer, MSD, Genesis and Sanofi. Christodoulou C. serves in a consultant/advisory role to Roche Hellas. Friberg L.E. serves in a consultant/advisory role to Genentech, Inc. (not on bevacizumab). One Ph.D. candidate from her research group, who is not involved in the current study, has received research funding from Genentech, Inc. Panoilia E., Schindler E., Samantas E., Aravantinos G., Patrinos G.P. and Sivolapenko G. declare no conflict of interests.

Open Access This article is distributed under the terms of the Creative Commons Attribution License which permits any use, distribution, and reproduction in any medium, provided the original author(s) and the source are credited.

\section{References}

1. Avastin-H/C/000582-II/65 (2014) European Public Assessment Report. The European Medicines Agency. http://www.ema.europa. eu/ema/index.jsp?curl=pages/medicines/human/medicines/000582/ human_med_000663.jsp\&murl=menus/medicines/medicines.jsp\& mid=WC0b01ac058001d125. Accessed 20 Aug 2014

2. Final Labeling Text, BLA 125085 (2013) Center for Drug Evaluation and Research. U.S. Food and Drug Administration. http://www.accessdata.fda.gov/drugsatfda_docs/ label/2013/125085s285lbl.pdf. Accessed 20 Aug 2014

3. Avastin ${ }^{\circledR}$ (2014) Genentech USA, Inc. http://www.avastin.com/ patient?cid=gne_WE_00000083. Accessed 20 Aug 2014

4. Parikh SS, Mehta HH, Desai BI (2012) Advances in development of bevacizumab, a humanized antiangiogenic therapeutic monoclonal antibody targeting VEGF in cancer cells. Int J Pharm Biomed Sci 3:155-163

5. Tabrizi M, Roskos LK (2006) Exposure-response relationships for therapeutic biologic products. In: Meibohm B (ed) Pharmacokinetics and pharmacodynamics of biotech drugs. Principles and case studies in drug development. WILEY-VCH Verlag GmbH \& Co. KGaA, Weinheim, pp 295-327

6. Gerber HP, Ferrara N (2005) Pharmacology and pharmacodynamics of bevacizumab as monotherapy or in combination with cytotoxic therapy in preclinical studies. Cancer Res 65:671-680

7. Pavlidis ET, Pavlidis TE (2013) Role of bevacizumab in colorectal cancer growth and its adverse effects: a review. World J Gastroenterol 19:5051-5060

8. Byrne AM, Bouchier-Hayes DJ, Harmey JH (2005) Angiogenic and cell survival functions of vascular endothelial growth factor (VEGF). J Cell Mol Med 9:777-794

9. Kemik O, Kemik AS, Sümer A, Purisa S, Dulger AC, Begenik H, Hasirci İ, Tuzun S (2011) Preoperative vascular endothelial growth factor levels as a prognostic marker for stage II or III colorectal cancer patients. Cancer Growth Metastasis 4:25-32
10. De Vita F, Orditura M, Lieto E, Infusino S, Morgillo F, Martinelli E, Castellano P, Romano C, Ciardiello F, Catalano G, Pignatelli C, Galizia G (2004) Elevated perioperative serum vascular endothelial growth factor levels in patients with colon carcinoma. Cancer 100:270-278

11. Fujisaki K, Mitsuyama K, Toyonaga A, Matsuo K, Tanikawa K (1998) Circulating vascular endothelial growth factor in patients with colorectal cancer. Am J Gastroenterol 93:249-252

12. Kazazi-Hyseni F, Beijnen JH, Schellens JHM (2010) Bevacizumab. Oncologist 15:819-825

13. Kim JG, Chae YS, Sohn SK, Cho YY, Moon JH, Park JY, Jeon SW, Lee IT, Choi GS, Jun SH (2008) Vascular endothelial growth factor gene polymorphisms associated with prognosis for patients with colorectal cancer. Clin Cancer Res 14:62-66

14. Stevens A, Soden J, Brenchley PE, Ralph S, Ray DW (2003) Haplotype analysis of the polymorphic human vascular endothelial growth factor gene promoter. Cancer Res 63:812-816

15. DeMichele A, Fox KR (2009) Optimizing outcomes with bevacizumab by better targeting patients and tumors. Oncology 23:339-340

16. Glassman PM, Balthasar JP (2014) Mechanistic considerations for the use of monoclonal antibodies for cancer therapy. Cancer Biol Med 11:20-33

17. Dostalek M, Gardner I, Gurbaxani BM, Rose RH, Chetty M (2013) Pharmacokinetics, pharmacodynamics and physiologically-based pharmacokinetic modelling of monoclonal antibodies. Clin Pharmacokinet 52:83-124

18. Bender BC, Schindler E, Friberg LE (2013) Population pharmacokinetic pharmacodynamic modelling in oncology: a tool for predicting clinical response. Br J Clin Pharmacol. doi:10.1111/ bcp. 12258

19. Mould DR, Upton RN (2012) Basic concepts in population modeling, simulation, and model-based drug development. CPT Pharmacomet Syst Pharmacol. doi:10.1038/psp.2012.4

20. Mould DR, Sweeney KR (2007) The pharmacokinetics and pharmacodynamics of monoclonal antibodies-mechanistic modeling applied to drug development. Curr Opin Drug Discov Dev 10:84-96

21. Ma P (2012) Theoretical considerations of target-mediated drug disposition models: simplifications and approximations. Pharm Res 29:866-882

22. Gibiansky L, Gibiansky E (2010) Target-mediated drug disposition model for drugs that bind to more than one target. J Pharmacokinet Pharmacodyn 37:323-346

23. Gibiansky L, Gibiansky E (2009) Target-mediated drug disposition model: approximations, identifiability of model parameters and applications to the population pharmacokinetic-pharmacodynamic modeling of biologics. Expert Opin Drug Metab Toxicol 5:803-812

24. Gibiansky L, Gibiansky E, Kakkar T, Ma P (2008) Approximations of the target-mediated drug disposition model and identifiability of model parameters. J Pharmacokinet Pharmacodyn 35:573-591

25. Turner DC, Navid F, Daw NC, Mao S, Wu J, Santana VM, Neel M, Rao B, Willert JR, Loeb DM, Harstead KE, Throm SL, Freeman BB, Stewart CF (2014) Population pharmacokinetics of bevacizumab in children with osteosarcoma: implications for dosing. Clin Cancer Res 20:2783-2792

26. Lu J, Bruno R, Eppler S, Novotny W, Lum B, Gaudreault J (2008) Clinical pharmacokinetics of bevacizumab in patients with solid tumors. Cancer Chemother Pharmacol 62:779-786

27. Rocchetti M, Germani M, Del Bene F, Poggesi I, Magni P, Pesenti E, De Nicolao G (2013) Predictive pharmacokineticpharmacodynamic modeling of tumor growth after administration of an anti-angiogenic agent, bevacizumab, as single-agent 
and combination therapy in tumor xenografts. Cancer Chemother Pharmacol 71:1147-1157

28. Watanabe T, Kobunai T, Yamamoto $\mathrm{Y}$, Matsuda $\mathrm{K}$, Ishihara $\mathrm{S}$, Nozawa K, Iinuma H, Ikeuchi H (2011) Gene expression of vascular endothelial growth factor A, thymidylate synthase, and tissue inhibitor of metalloproteinase 3 in prediction of response to bevacizumab treatment in colorectal cancer patients. Dis Colon Rectum 54:1026-1035

29. Loupakis F, Cremolini C, Fioravanti A, Orlandi P, Salvatore L, Masi G, Di Desidero T, Canu B, Schirripa M, Frumento P, Di Paolo A, Danesi R, Falcone A, Bocci G (2011) Pharmacodynamic and pharmacogenetic angiogenesis-related markers of first-line FOLFOXIRI plus bevacizumab schedule in metastatic colorectal cancer. Br J Cancer 104:1262-1269

30. Eisenhauer EA, Therasse P, Bogaerts J, Schwartz LH, Sargent D, Ford R, Dancey J, Arbuck S, Gwyther S, Mooney M, Rubinstein L, Shankar L, Dodd L, Kaplan R, Lacombe D, Verweij J (2009) New response evaluation criteria in solid tumours: revised RECIST guide-line (version 1.1). Eur J Cancer 45:228-247

31. Ternant D, Cézé N, Lecomte T, Degenne D, Duveau AC, Watier H, Dorval E, Paintaud G (2010) An enzyme-linked immunosorbent assay to study bevacizumab pharmacokinetics. Ther Drug Monit 32:647-652

32. Quantikine ${ }^{\circledR}$ human VEGF immunoassay (2014) R\&D Systems Inc., Minneapolis, USA. http://www.rndsystems.com/pdf/dve00. pdf. Accessed 10 Sept 2014

33. Shendure JA, Porreca GJ, Church GM, Gardner AF, Hendrickson CL, Kieleczawa J, Slatko BE (2011) Overview of DNA sequencing strategies. Curr Protoc Mol Biol 96:7.1.1-7.1.23

34. DNA sequencing by capillary electrophoresis (2009) Applied Biosystems chemistry guide. https://www3.appliedbiosystems. com/cms/groups/mcb_support/documents/generaldocuments/ cms_041003.pdf. Accessed 11 Sept 2014

35. Jonsson EN, Karlsson MO (1999) Xpose-an S-PLUS based population pharmacokinetic/pharmacodynamic model building aid for NONMEM. Comput Methods Programs Biomed 58:51-64

36. Lindbom L, Pihlgren P, Jonsson EN (2005) PsN-Toolkit-a collection of computer intensive statistical methods for non-linear mixed effect modeling using NONMEM. Comput Methods Programs Biomed 79:241-257

37. Savic RM, Karlsson MO (2009) Importance of shrinkage in empirical Bayes estimates for diagnostics: problems and solutions. AAPS J 11:558-569

38. Holford NH (1996) A size standard for pharmacokinetics. Clin Pharmacokinet 30:329-332

39. Wählby U, Jonsson EN, Karlsson MO (2001) Assessment of actual significance levels for covariate effects in NONMEM. J Pharmacokinet Pharmacodyn 28:231-252

40. Bergstrand M, Hooker AC, Wallin JE, Karlsson MO (2011) Prediction-corrected visual predictive checks for diagnosing nonlinear mixed-effects models. AAPS J 13:143-151
41. Keizer RJ, Karlsson MO, Hooker A (2013) Modeling and simulation workbench for NONMEM: tutorial on Pirana, PsN, and Xpose. CPT Pharmacomet Syst Pharmacol. doi:10.1038/ psp.2013.24

42. McLeay SC, Morrish GA, Kirkpatrick CM, Green B (2012) The relationship between drug clearance and body size: systematic review and meta-analysis of the literature published from 2000 to 2007. Clin Pharmacokinet 51:319-330

43. Papadopoulos N, Martin J, Ruan Q, Rafique A, Rosconi MP, Shi E, Pyles EA, Yancopoulos GD, Stahl N, Wiegand SJ (2012) Binding and neutralization of vascular endothelial growth factor (VEGF) and related ligands by VEGF Trap, ranibizumab and bevacizumab. Angiogenesis 15:171-185. doi:10.1007/ s10456-011-9249-6

44. Chen Y, Wiesmann C, Fuh G, Li B, Christinger HW, McKay P, de Vos AM, Lowman HB (1999) Selection and analysis of an optimized anti-VEGF antibody: crystal structure of an affinitymatured Fab in complex with antigen. J Mol Biol 293:865-881

45. Gaudreault J, Greig G, Cosson V, Gupta M, Jumbe N, Hooker AC (2008) Population pharmacokinetics of bevacizumab. J Clin Oncol 26:abstr 14570

46. Gordon MS, Margolin K, Talpaz M, Sledge GW Jr, Holmgren E, Benjamin R, Stalter S, Shak S, Adelman D (2001) Phase I safety and pharmacokinetic study of recombinant human anti-vascular endothelial growth factor in patients with advanced cancer. J Clin Oncol 19:843-850

47. Min BS, Kim NK, Jeong HC, Chung HC (2012) High levels of serum VEGF and TIMP-1 are correlated with colon cancer liver metastasis and intrahepatic recurrence after liver resection. Oncol Lett 4:123-130

48. Hansson EK, Amantea MA, Westwood P, Milligan PA, Houk BE, French J, Karlsson MO, Friberg LE (2013) PKPD modeling of VEGF, sVEGFR-2, sVEGFR-3, and sKIT as predictors of tumor dynamics and overall survival following sunitinib treatment in GIST. CPT Pharmacomet Syst Pharmacol. doi:10.1038/psp.2013.61

49. Stefanini MO, Wu FT, Mac Gabhann F, Popel AS (2010) Increase of plasma VEGF after intravenous administration of bevacizumab is predicted by a pharmacokinetic model. Cancer Res 70:9886-9894

50. Hsei V, Deguzman GG, Nixon A, Gaudreault J (2002) Complexation of VEGF with bevacizumab decreases VEGF clearance in rats. Pharm Res 19:1753-1756

51. Rudge JS, Holash J, Hylton D, Russell M, Jiang S, Leidich R, Papadopoulos N, Pyles EA, Torri A, Wiegand SJ, Thurston G, Stahl N, Yancopoulos GD (2007) VEGF Trap complex formation measures production rates of VEGF, providing a biomarker for predicting efficacious angiogenic blockade. Proc Natl Acad Sci USA 104:18363-18370

52. Verheul HM, Lolkema MP, Qian DZ, Hilkes YH, Liapi E, Akkerman JW, Pili R, Voest EE (2007) Platelets take up the monoclonal antibody bevacizumab. Clin Cancer Res 13:5341-5347 